\title{
Validation expérimentale et numérique d'un modèle pour l'étude de l'emboutissage de tôles minces
}

\author{
Mohsen Akrout ${ }^{1, a}$ et Chineb Chaker ${ }^{2}$ \\ 1 École Nationale des Ingénieurs de Sfax (ENIS), Département de Mécanique, Unité de Mécanique, Modélisation et Production \\ (U2MP), Route de Soukra km 4, BP W 3038, Sfax, Tunisie \\ 2 École Nationale des Ingénieurs de Sfax (ENIS), Laboratoire des Systèmes Électro-Mécaniques (LASEM), Route de Soukra \\ km 4, BP W 3038, Sfax, Tunisie
}

Reçu le 4 décembre 2002, accepté le 8 juillet 2005

\begin{abstract}
Résumé - Dans cet article, on présente d'abord la validation expérimentale de l'aspect frottement du logiciel «PROFIL » d'emboutissage de tôles minces. Cet aspect a été validé par des essais expérimentaux réalisés sur la machine d'emboutissage TESTOLUB du C.E.T.I.M. La modélisation du frottement par un effort de retenue appliqué au bout de la tôle s'est avérée comme une solution simple et efficace. Ensuite, on teste numériquement ce logiciel par le code de calcul d'éléments finis «ABAQUS » en choisissant des éléments quadratiques afin de tenir compte de la variation d'épaisseur de la tôle au cours de l'opération d'emboutissage.
\end{abstract}

Mots clés : Emboutissage / tôle mince / modélisation / frottement

\begin{abstract}
Experimental and numerical validation of a model for the study of thin sheet stamping. In this article, we present initially the experimental validation of the friction aspect of the software "PROFIL" of thin sheet stamping. This aspect was validated by experimental tests carried out on the machine of stamping TESTOLUB of the C.E.T.I.M. The modeling of friction by an effort of reserve applied at the end of sheet appears as a simple and effective solution. Then, we test this software numerically by the computer code of finite elements "ABAQUS" by choosing quadratic elements in order to take account of the variation thickness of sheet during the stamping operation.
\end{abstract}

Key words: stamping / thin sheet / modeling / friction

\section{Introduction}

L'emboutissage des tôles minces est un procédé de mise en forme couramment utilisé dans l'industrie. Il consiste à déformer plastiquement des tôles minces afin d'obtenir des pièces de formes complexes. Cependant, cette technique est confrontée au problème du retour élastique dû à la restitution de l'énergie élastique emmagasinée au cours de la phase de chargement, ce qui conduit à un changement de géométrie de la pièce emboutie [1].

Étant donné la complexité et le coût élevé du processus d'emboutissage, les essais expérimentaux ne peuvent pas être poussés sans limitations matérielles et technologiques, d'où la nécessité de logiciels informatiques pouvant gérer cette technique et maîtrisant le phénomène du retour élastique. Une simulation numérique d'un tel

\footnotetext{
a Auteur correspondant : mohsenakrout@yahoo.fr
}

procédé, que ce soit par une méthode analytique $[2,3]$ ou par une méthode des éléments finis $[4,5]$, sera très utile pour la compréhension du phénomène et la maîtrise de ses différents paramètres technologiques [6]. Grâce à ses performances, la méthode des éléments finis semble la plus adéquate pour la simulation numérique des processus de mise en forme $[7,8]$.

À partir de ce besoin, le logiciel «PROFIL» d'emboutissage de tôles minces a été élaboré au Laboratoire de Mécanique des Solides de l'I.N.S.A. de Lyon et ensuite testé et validé par des essais expérimentaux et aussi comparé au code de calcul «ABAQUS».

\section{Présentation du logiciel «PROFIL»}

«PROFIL $»$ est un logiciel utilisant des éléments finis de type coque en déformation plane de continuité $C_{0}$ 
(« Mindlin ») [9]. Il permet de simuler l'emboutissage des tôles minces et de prévoir leur retour élastique [10]. La phase d'emboutissage consiste à faire descendre le poinçon qui vient plier la tôle maintenue entre la matrice et le serre-flan. La phase du retour élastique concerne le retrait des outils et l'extraction de la tôle emboutie.

Le calcul de ce retour par le logiciel «PROFIL» est obtenu en décrémentant les efforts extérieurs atteints en fin d'emboutissage, la tôle est ainsi libérée des efforts dus au contact avec les outils. Le retour élastique prend fin lorsque toutes les forces de contact sont annulées [11].

\section{Validation expérimentale du frottement}

Afin de valider l'aspect frottement [12] dans le logiciel «PROFIL», on se propose de l'utiliser pour comparer ses résultats avec ceux obtenus expérimentalement grâce à un essai d'emboutissage réalisé au C.E.T.I.M.

\subsection{Description de l'essai TESTOLUB du C.E.T.I.M.}

Afin de comparer le pouvoir lubrifiant de différentes huiles, le C.E.T.I.M. a mis au point un essai d'emboutissage de tôle appelé TESTOLUB (Fig. 1).

Cet essai consiste à effectuer, sur un outillage de pliage en U, le formage d'une tôle recouverte du lubrifiant à caractériser en augmentant progressivement l'effort du serre-flan $F_{\mathrm{SF}}$ jusqu'à atteindre un effort poinçon $F_{\mathrm{p}}$ prédéterminé : le lubrifiant sera d'autant plus efficient que l'effort serre-flan à l'arrêt sera élevé. On définit ainsi le coefficient de frottement apparent :

$$
A=\frac{F_{\mathrm{p}}}{F_{\mathrm{SF}}}
$$

Ce coefficient caractérise le pouvoir lubrifiant de l'huile utilisée et permet de la classer de I à IV (Tab. 1) dans l'ordre croissant du pouvoir lubrifiant (Fig. 2).

\subsubsection{Conditions opératoires}

Pour fixer la tôle, on établit au préalable une pression de 60 bars entre le serre-flan et la matrice de rayon $7 \mathrm{~mm}$. Le jeu radial poinçon-matrice est égal à la moitié de l'épaisseur de la tôle. Le poinçon commence alors à monter jusqu'à ce qu'il atteigne l'une des pressions suivantes selon le choix de l'opérateur : 25; 50 ou 100 bars. On lit alors la profondeur du pliage et la pression du serre-flan (Fig. 3).

La course totale possible du poinçon est $200 \mathrm{~mm}$. Si la pression sélectionnée n'est pas atteinte avant la fin de cette course, l'essai s'arrête. Il faudra alors passer à la pression d'arrêt inférieure. La longueur du flan est déterminée de telle façon qu'en fin d'essai il reste toujours un bout de la tôle sous le serre-flan.

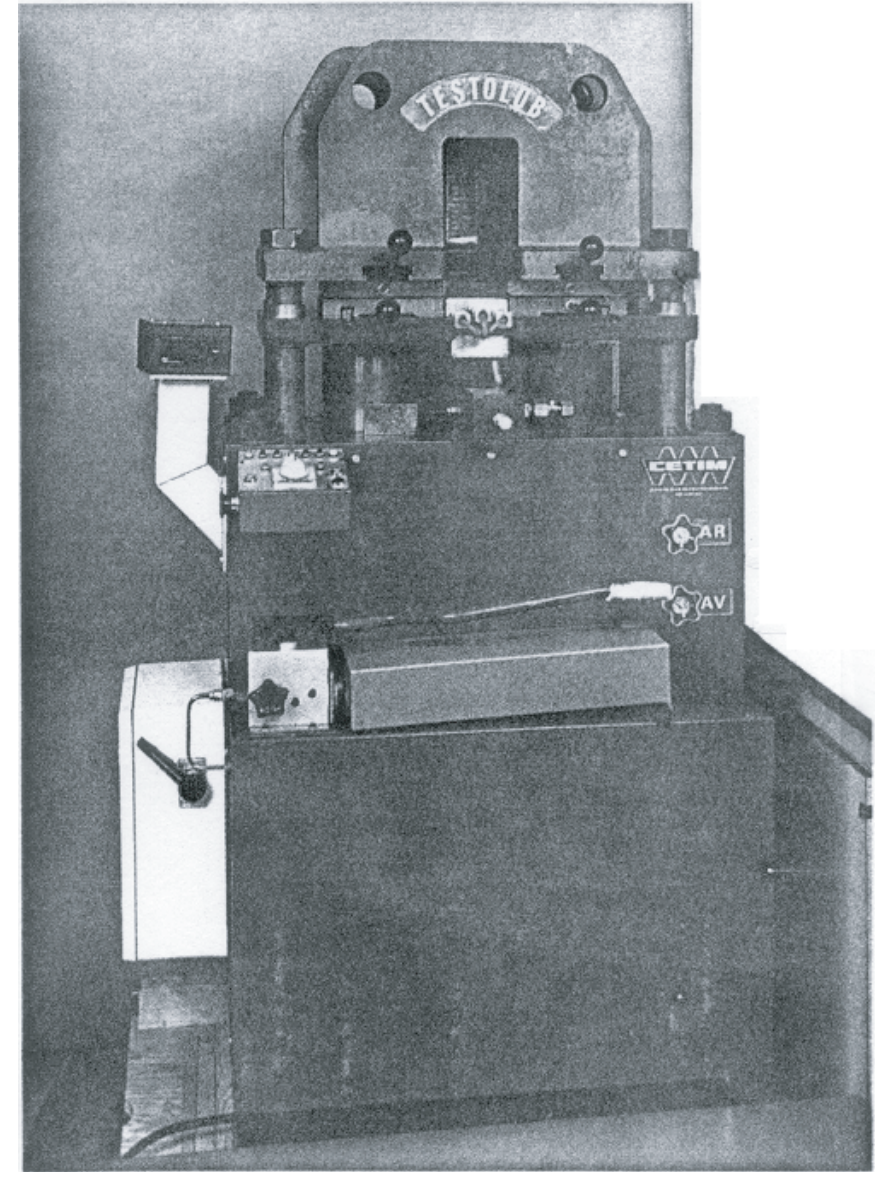

Fig. 1. Machine d'emboutissage TESTOLUB.

\subsubsection{Relation effort serre-flan - Déplacement poinçon}

Au cours d'un essai TESTOLUB, l'effort $F_{\mathrm{SF}}$ d'un seul serre-flan croît linéairement avec le déplacement du poinçon $h$ suivant la loi suivante :

$$
F_{\mathrm{SF}}=0,247 \cdot h+48
$$

\subsubsection{Effort du serre-flan par unité de largeur}

La largeur de la tôle étant égale à $150 \mathrm{~mm}$, l'effort du serre-flan par unité de largeur s'écrit alors :

$$
F_{\mathrm{SF}}=0,165 \cdot h+32
$$

\subsubsection{Relation effort serre-flan - Pression serre-flan}

La surface de contact entre la tôle et un seul serre-flan est de $80 \mathrm{~cm}^{2}$. La relation entre l'effort d'un seul serre-flan et sa pression est :

$$
F_{\mathrm{SF}}=0,8 \cdot P_{\mathrm{SF}}
$$


Tableau 1. Classification du pouvoir lubrifiant de l'huile.

\begin{tabular}{cccc}
\hline $\begin{array}{c}\text { Classe du } \\
\text { lubrifiant }\end{array}$ & $\begin{array}{c}\text { Effort poinçon } \\
F_{\mathrm{p}}(\mathrm{kN})\end{array}$ & $\begin{array}{c}\text { Hauteur du pliage } \\
h(\mathrm{~mm})\end{array}$ & Coefficient $A$ \\
\hline I & 50 & $<200$ & $0,258<A<0,530$ \\
II & 25 & $<200$ & $0,129<A<0,258$ \\
III & 12,5 & $<200$ & $0,064<A<0,129$ \\
IV & $<12,5$ & $=200$ & $A<0,064$ \\
\hline
\end{tabular}

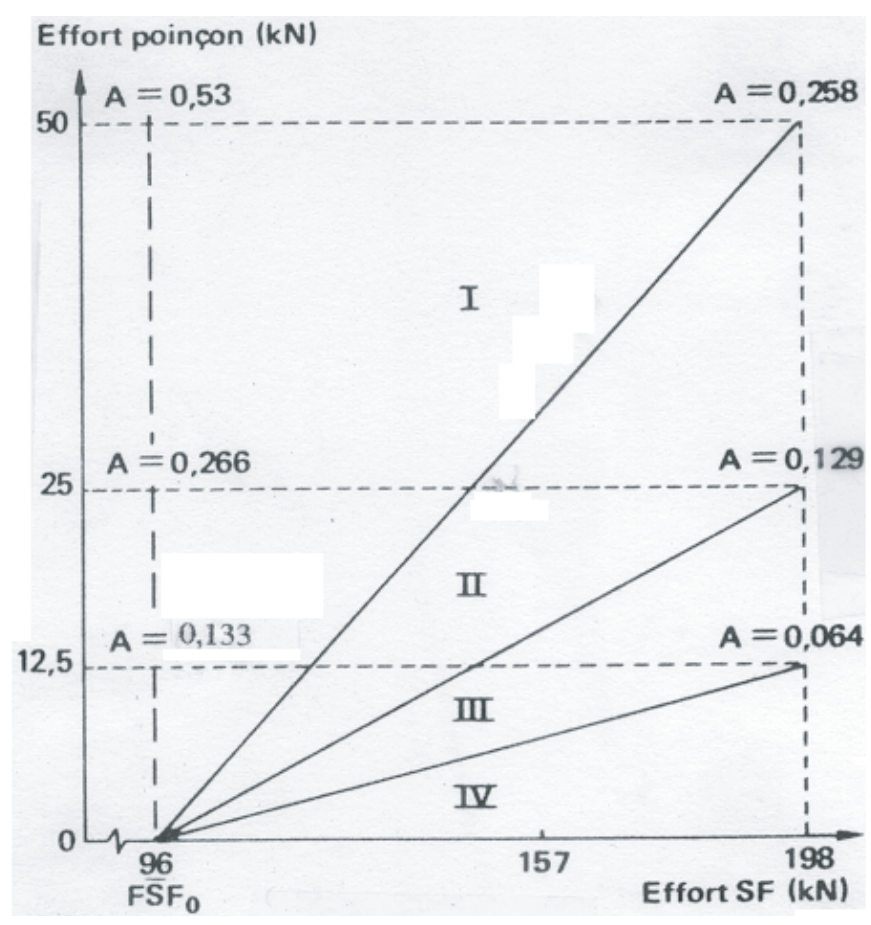

Fig. 2. Classification du pouvoir lubrifiant de l'huile.

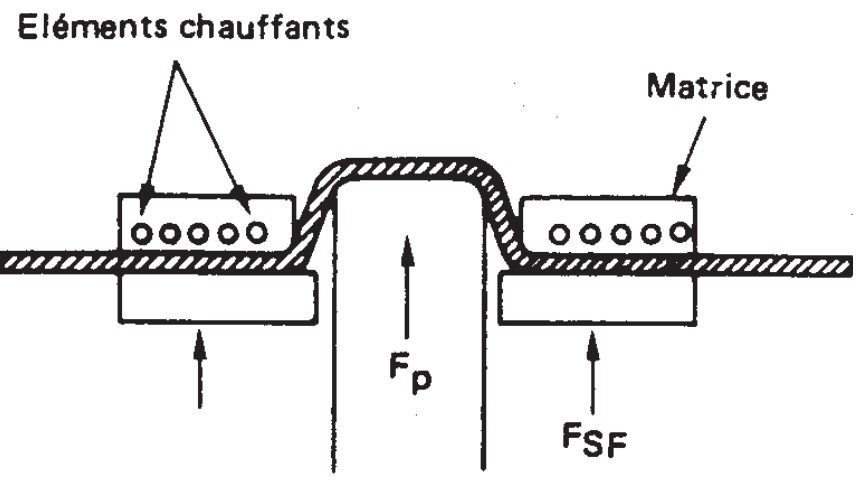

Fig. 3. Essai d'emboutissage TESTOLUB.

\subsubsection{Relation pression serre-flan - Déplacement poinçon}

La loi de variation de la pression du serre-flan en fonction du déplacement du poinçon est :

$$
P_{\mathrm{SF}}=0,309 \cdot h+60
$$

\subsubsection{Caractéristiques de l'essai}

- Matériau : Tôle XES

- Dimensions de la tôle : $900 \times 150 \times 0,8 \mathrm{~mm}$

- Loi d'écrouissage de SWIFT : $\sigma=B .\left(C+\varepsilon^{p}\right)^{n}$ avec $B=559 \mathrm{MPa}$

$$
\begin{aligned}
& C=2,7 \times 10^{-3} \\
& n=0,222
\end{aligned}
$$

- Limite élastique : $\sigma_{\mathrm{e}}=150 \mathrm{MPa}$

- Module de Young : $E=200000 \mathrm{MPa}$

- Coefficient de Poisson : $\nu=0,3$

- Jeu (poinçon-matrice) /épaisseur $=1,5$

- Longueur de la partie plate du poinçon : $L_{\mathrm{p}}=43 \mathrm{~mm}$

- Rayon du poinçon : $R_{\mathrm{p}}=7 \mathrm{~mm}$

- Rayon de la matrice : $R_{\mathrm{m}}=7 \mathrm{~mm}$

- Profondeur des emboutis $=50 ; 100 ; 150$ et $200 \mathrm{~mm}$

- Vitesse de déplacement du poinçon $=115 \mathrm{~mm} \cdot \mathrm{min}^{-1}$

- Lubrifiant : huile ILOFORME FS 228.

\subsection{Modélisation du frottement dans «PROFIL »}

Le lubrifiant choisi pour cet essai est une huile du type ILOFORME FS 228, elle introduit un frottement dont le coefficient est égal à 0,183 .

Dans un frottement de Coulomb, l'effort tangentiel $F_{\mathrm{t}}$ est égal au produit de l'effort normal $F_{\mathrm{n}}$ par le coefficient de frottement $\mu$

$$
\left|F_{\mathrm{t}}\right|=\mu \cdot\left|F_{\mathrm{n}}\right|
$$

Pour tenir compte du serre-flan dans cette version du programme «PROFIL», un effort de retenue est appliqué au bout de la tôle : le serre-flan n'impose ici que des conditions géométriques [13]. Cet effort, dû au frottement engendré par un seul serre-flan, croît linéairement avec le déplacement du poinçon suivant la relation suivante :

$$
F_{\text {retenue }}=\mu \cdot F_{\mathrm{SF}}
$$

d'où :

$$
F_{\text {retenue }}=44,46 \cdot h+8640
$$

Cet emboutissage a été simulé à l'aide du programme «PROFIL $»$ pour chacune des profondeurs suivantes : 50 ; $100 ; 150$ et $200 \mathrm{~mm}$. Pour chaque profondeur, on libère la tôle de ses forces de contact pour avoir sa déformée après retour élastique. On prendra comme critères de comparaison l'effort du poinçon en fonction de son déplacement et l'inclinaison $\alpha$ que fait la partie externe non déformée avec l'horizontale (Fig. 4).

On obtient ainsi le tableau des résultats suivant (Tab. 2) et on trace la variation de l'inclinaison $\alpha$ et de 


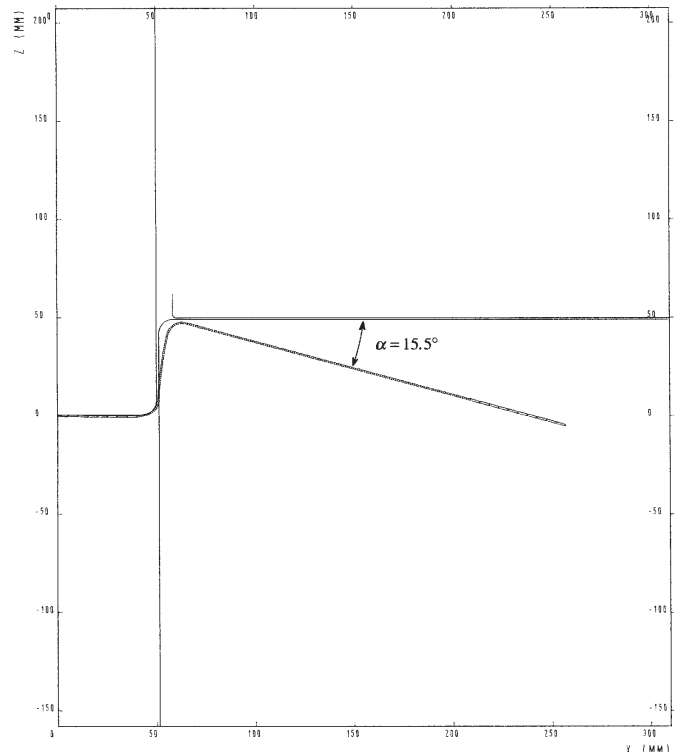

(a)

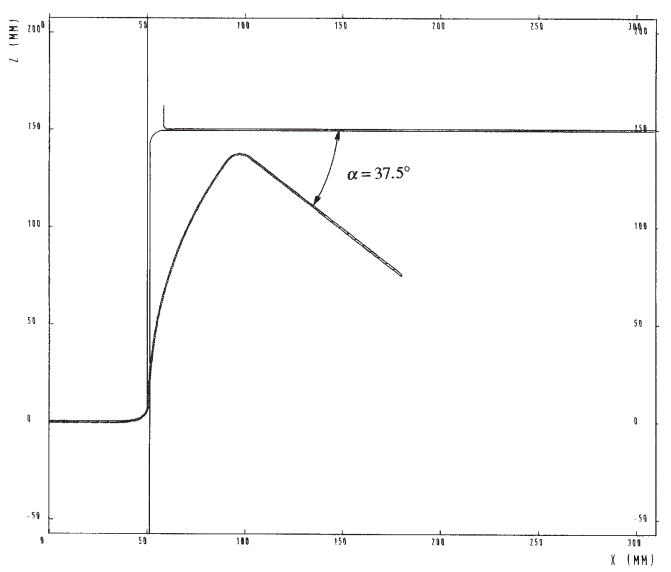

(c)

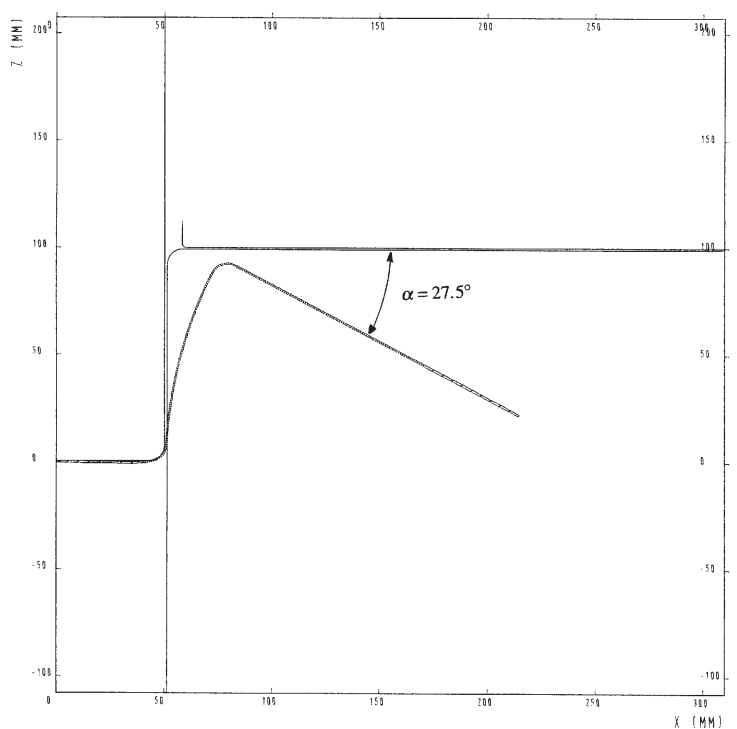

(b)

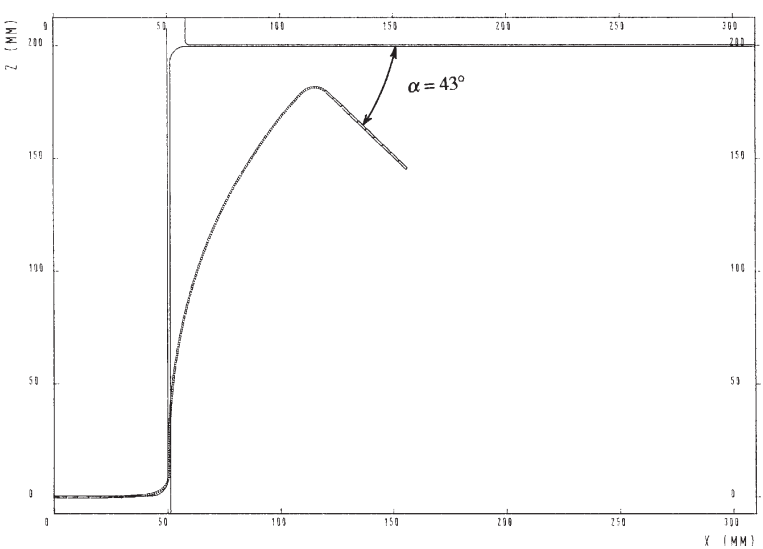

(d)

Fig. 4. Retour élastique pour différentes profondeurs d'emboutissage, (a) $h=50 \mathrm{~mm}$, (b) $h=100 \mathrm{~mm}$, (c) $h=150 \mathrm{~mm}$, (d) $h=200 \mathrm{~mm}$.

Tableau 2. Résultats des essais avec TESTOLUB et «PROFIL ».

\begin{tabular}{ccccc}
\hline Profondeur $h(\mathrm{~mm})$ & 50 & 100 & 150 & 200 \\
\hline$\alpha_{\text {TESTOLUB }}\left({ }^{\circ}\right)$ & 15 & 25 & 36,5 & 41 \\
\hline$\alpha_{\text {PROFIL }}\left(^{\circ}\right)$ & 15,5 & 27,5 & 37,5 & 43 \\
\hline$F_{\text {TESTOLUB }}(\mathrm{daN} / \mathrm{mm})$ & 10,5 & 16,5 & 21,3 & 25,2 \\
\hline$F_{\text {PROFIL }}(\mathrm{daN} / \mathrm{mm})$ & 9,6 & 14,2 & 20,8 & 23,5 \\
\hline
\end{tabular}

l'effort $F$ en fonction de la profondeur d'emboutissage $h$ (Fig. 5).

En comparant les résultats expérimentaux de l'essai TESTOLUB avec ceux de «PROFIL», on constate que ces derniers sont très satisfaisants. Ainsi, la modélisation du frottement dû au serre-flan par un effort de retenue appliqué au bout de la tôle apparaît comme une solution simple et efficace.

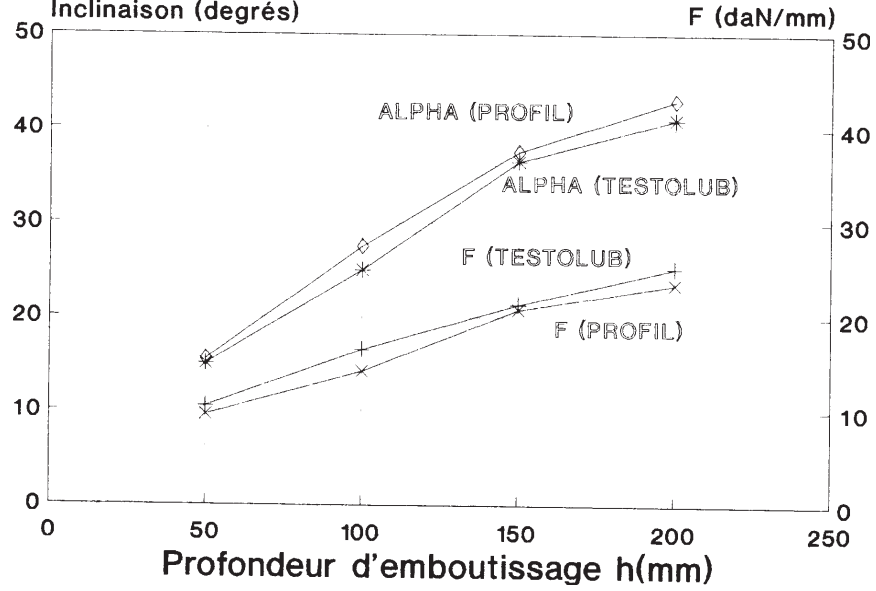

Fig. 5. Variation de l'inclinaison $\alpha$ et de l'effort d'emboutissage $F$ en fonction de la profondeur $h$. 


\section{Validation numérique par le code de calcul ABAQUS »}

Un autre outil de calcul a contribué à la validation de «PROFIL» [14] : c'est le code de calcul d'éléments finis «ABAUS». Afin de comparer ces deux logiciels, on simulera l'emboutissage d'un demi-flan en acier XE280D d'épaisseur $1 \mathrm{~mm}$, de largeur $50 \mathrm{~mm}$ et de longueur $100 \mathrm{~mm}$ (l'autre moitié étant obtenue par symétrie).

L'emboutissage s'effectue sur une profondeur de $45 \mathrm{~mm}$ avec un rayon d'entrée matrice de $5 \mathrm{~mm}$ et un rayon de poinçon de $13 \mathrm{~mm}$. Au cours de cette simulation, le coefficient de frottement sera égal à 0,125 .

\subsection{Simulation avec « ABAQUS »}

Pour simuler cet emboutissage avec le code de calcul «ABAQUS», il faut choisir des éléments bidimensionnels pour pouvoir tenir compte de l'épaisseur de la tôle.

Un premier calcul a été effectué avec des éléments CPE4 bidimensionnels linéaires à 4 nœuds, il s'est révélé insatisfaisant. En effet, ces éléments ayant uniquement des nœuds aux extrémités ne fournissent pas de bons résultats lorsqu'ils sont sollicités en flexion. Pour mieux résoudre ce problème, il faut avoir un nœud intermédiaire entre deux nouds aux extrémités, on utilisera donc des éléments à 8 nœuds (éléments quadratiques).

Dans cette étude, le flan est subdivisé en deux couches d'éléments CPE8 (élément bidimensionnel quadrangulaire à 8 nouds et à 9 points d'intégration), soit un maillage de 80 éléments (Fig. 6).

Le calcul nécessite alors 588 incréments (Fig. 7) et plus de 15 heures de temps CPU sur une station APOLLO 10000.

Avec le logiciel «ABAQUS» (Fig. 8), l'opération d'emboutissage se déroule en 4 étapes.

\section{$1^{\text {re }}$ étape : Descente du poinçon (Fig. 9)}

C'est la phase d'emboutissage, elle consiste à imposer un déplacement vertical au poinçon. La tôle est alors astreinte à se plier d'une part au rayon du poinçon et d'autre part au rayon de la matrice. Ce déplacement est appliqué en plusieurs pas. À chaque pas, le poinçon avance d'un incrément de déplacement fixé lors de la mise en données du problème. À l'extrémité gauche, la tôle est astreinte à subir un déplacement vertical sans, pour autant, être obligée à rester collée au poinçon, ce qui engendre un léger décollement entre le flan et le poinçon à cet endroit.

\section{$2^{e}$ étape : Remontée du poinçon (Fig. 10)}

Il s'agit là d'imposer au poinçon un déplacement opposé au précédent jusqu'au retour à sa position initiale. Cette étape déclenche la phase du retour élastique, elle est beaucoup plus rapide en temps de calcul que la précédente car la convergence du processus d'itération est vite atteinte et le programme n'est pas obligé de subdiviser

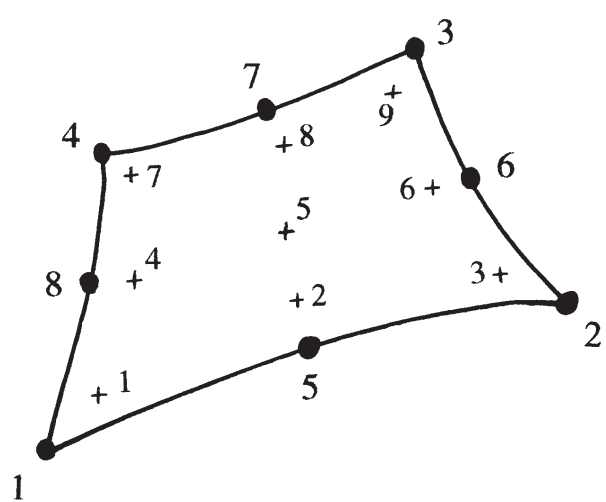

Fig. 6. Élément CPE8 (8 nœuds, 9 points d'intégration).

le pas d'incrémentation plusieurs fois pour éviter la divergence des calculs, chose qui n'était pas possible dans l'étape précédente.

\section{$3^{e}$ étape: Dégagement du serre-flan (Fig. 11)}

Cette étape consiste à soulever le serre-flan. Les efforts exercés par ce dernier sur la tôle sont alors annulés.

\section{$4^{e}$ étape : Dégagement de la matrice (Fig. 12)}

En retirant la matrice jusqu'à la fin du contact avec le flan, ce dernier est alors libéré de tous les efforts extérieurs. À la fin du retour élastique, la tôle n'est soumise qu'aux efforts intérieurs dus aux contraintes résiduelles dans le matériau.

\subsection{Simulation avec «PROFIL *}

Pour simuler cet emboutissage avec le logiciel « $P R O$ $F I L »$, le flan est subdivisé en 230 éléments (Fig. 13).

Pour résoudre cette modélisation, le logiciel « $P R O$ $F I L »$ nécessite environ 1 heure de temps CPU sur la station APOLLO 10000 et l'opération se déroule en 1954 incréments avec un pas de 0,025 mm (Fig. 14).

L'opération d'emboutissage se déroule en deux phases : une phase d'emboutissage (Fig. 15) et une phase de retour élastique (Fig. 16).

\section{3 Étude comparative}

En faisant une étude comparative entre les deux logiciels, le programme «PROFIL » a l'avantage d'être plus rapide en exécution et plus simple en utilisation.

Dans le code de calcul « $A B A Q U S$ », le retour élastique s'obtient par retrait des outils jusqu'à suppression de leur contact avec la tôle. Alors qu'avec le logiciel «PRO$F I L »$, le retour élastique peut être obtenu soit par décrémentation des efforts extérieurs atteints en fin d'emboutissage jusqu'à leur annulation, soit par retrait des outils jusqu'à suppression du contact.

Il faut noter aussi que le logiciel « $A B A Q U S »$, grâce à ses éléments quadratiques, tient compte du rebond qui se 

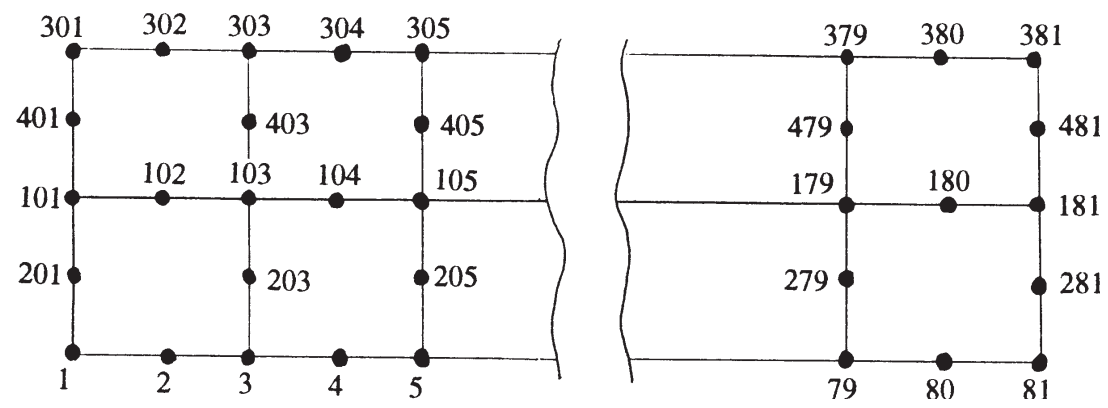

Fig. 7. Modélisation du flan par «ABAQUS».

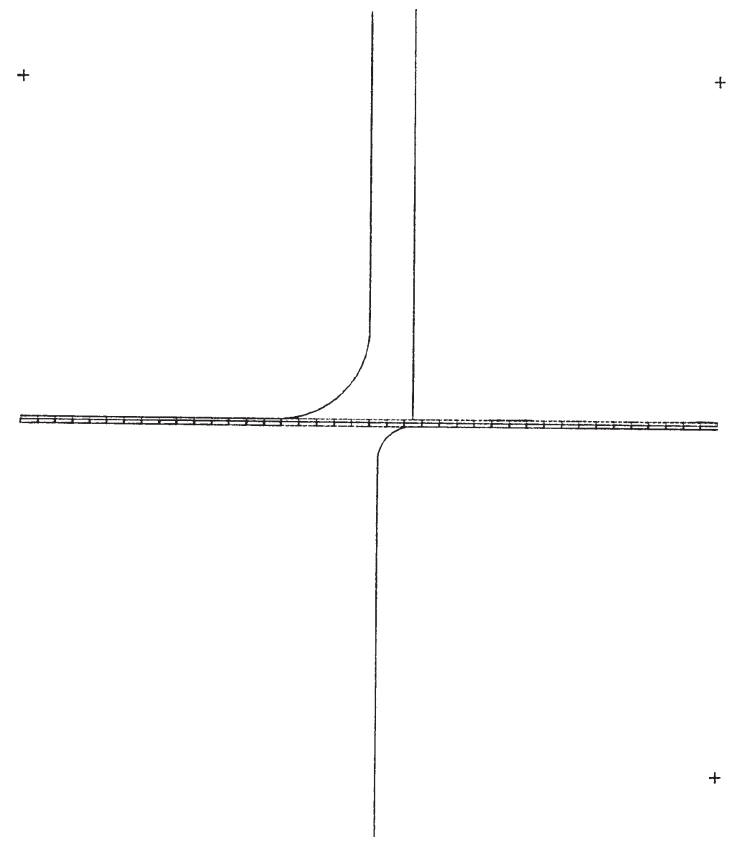

Fig. 8. Configuration initiale de la tôle avec « ABAQUS ».

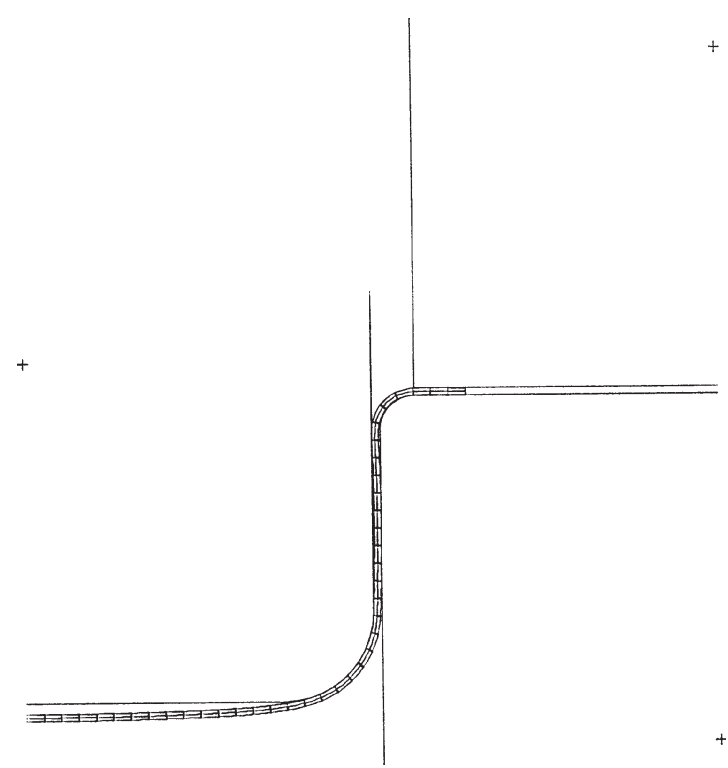

Fig. 9. Emboutissage de la tôle par «ABAQUS».

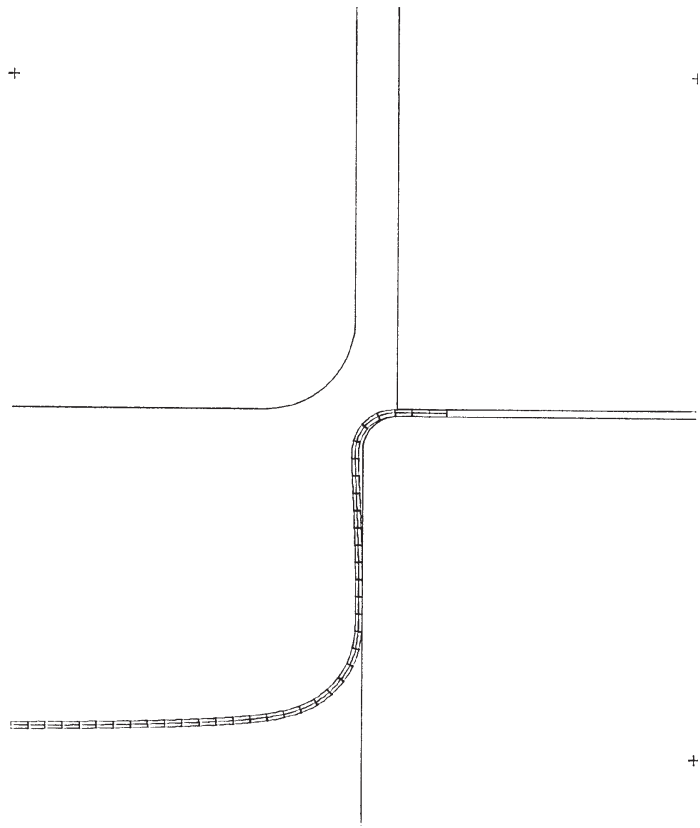

Fig. 10. Remontée du poinçon.

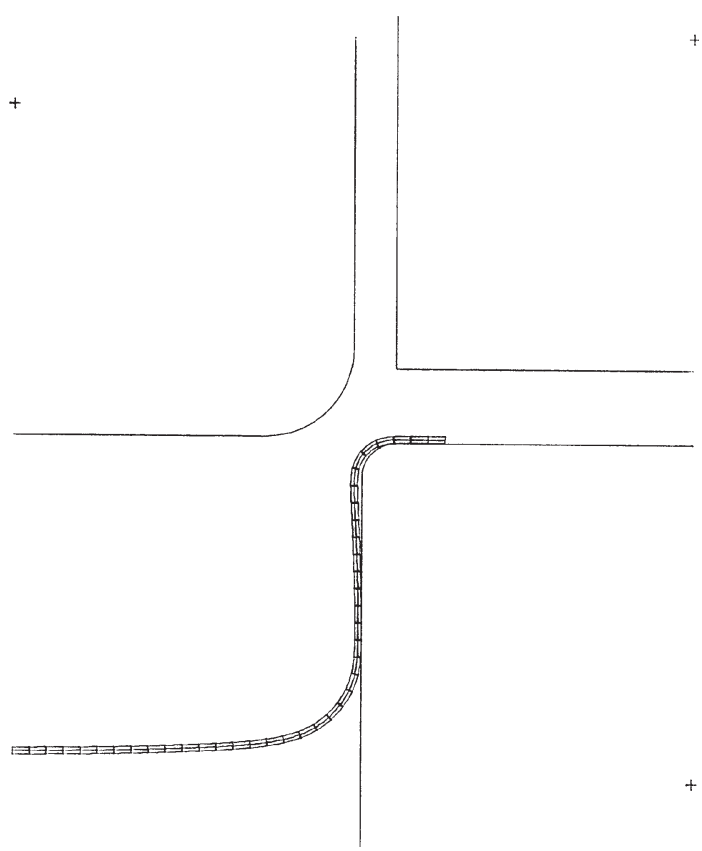

Fig. 11. Dégagement du serre-flan. 


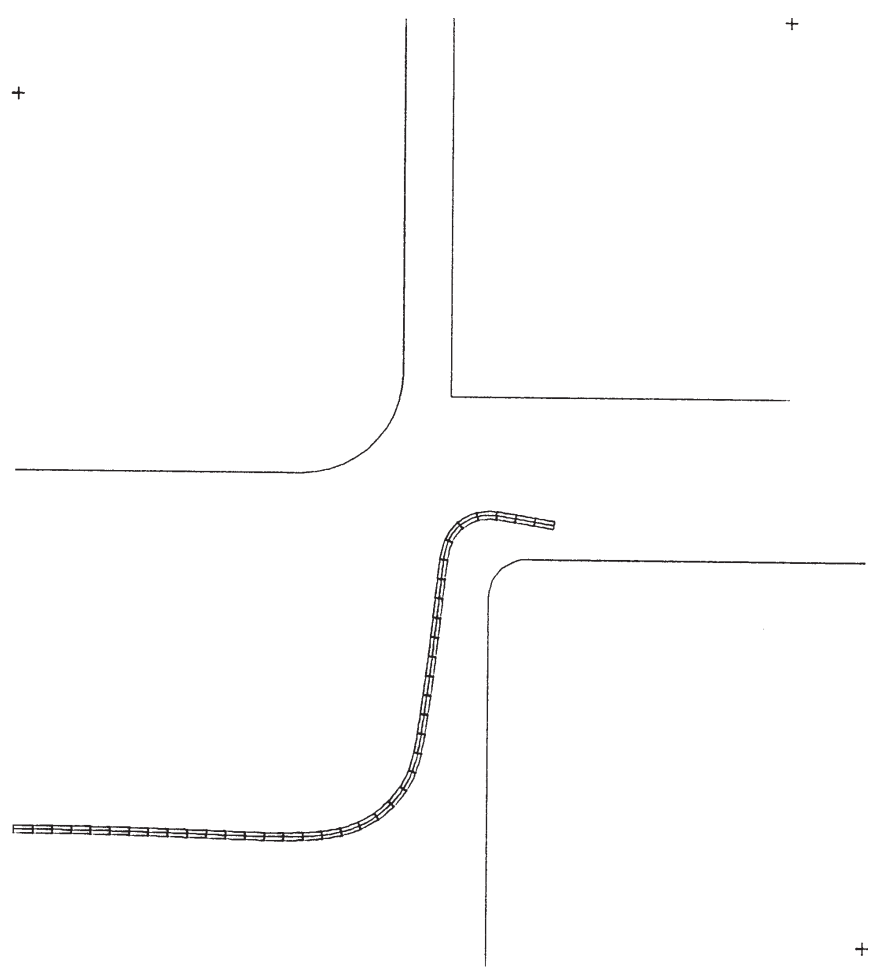

Fig. 12. Fin du retour élastique.

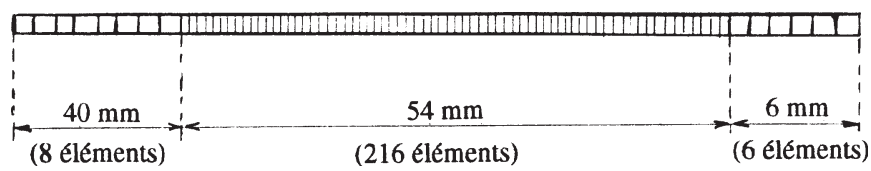

Fig. 13. Modélisation du flan par «PROFIL».

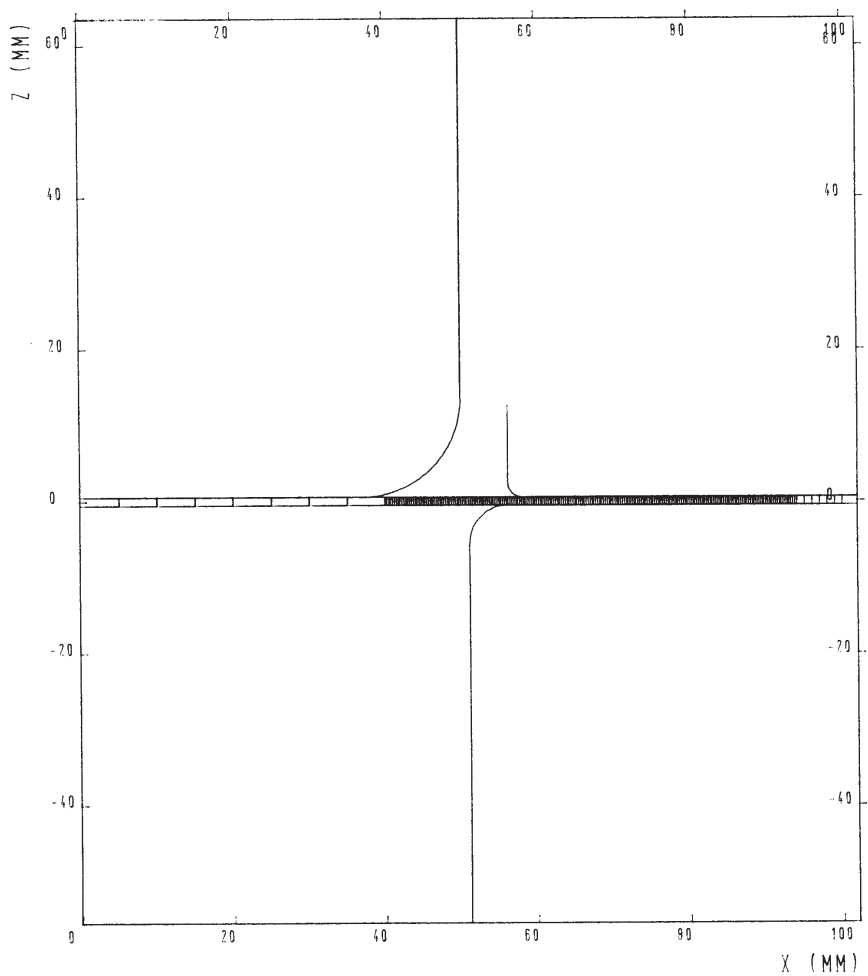

Fig. 14. Configuration initiale de la tôle avec «PROFIL».

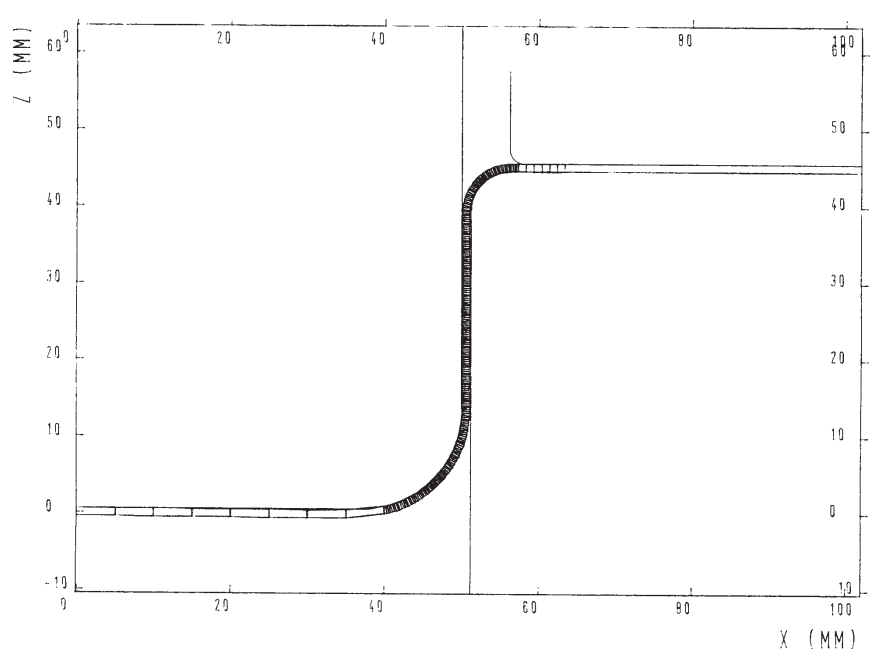

Fig. 15. Fin d'emboutissage.

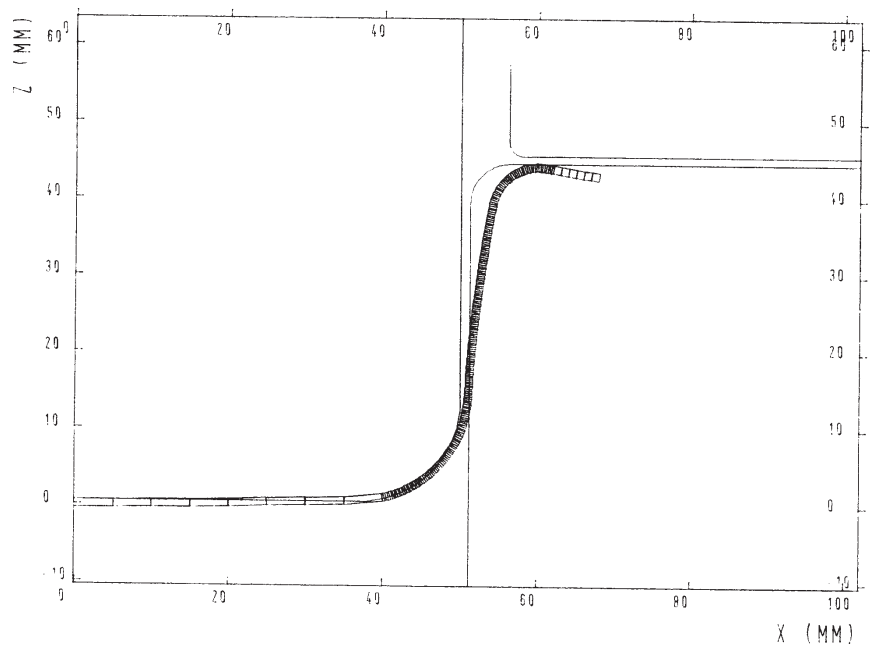

Fig. 16. Fin du retour élastique.

produit dans le mur entre le poinçon et la matrice tandis que le logiciel «PROFIL», avec ses éléments « poutres », ne peut pas en tenir compte.

Malgré cette différence, la comparaison des résultats des deux logiciels s'avère satisfaisante : les déformées obtenues avant et après retour élastique sont presque identiques $\left(\alpha_{\text {PROFIL }}=9,4^{\circ}\right.$ et $\left.\alpha_{\text {ABAQUS }}=8,8^{\circ}\right)$.

De même, le tracé de l'effort d'emboutissage $F$ en fonction de la profondeur $h$ (Fig. 17) montre que l'effort du poinçon au cours de la phase d'emboutissage est légèrement plus faible avec le logiciel «PROFIL» qu'avec le logiciel «ABAQUS» mais la différence reste petite (inférieure à $10 \%$ ).

Cette validation numérique avec le code de calcul «ABAQUS » aurait pu être poussée plus loin surtout pour étudier le cas d'embouti avec de faibles rayons d'entrée matrice présentant des phénomènes de rebond sur le mur. Malheureusement, ceci n'a pas été possible car ce code diverge tout le temps pour des rayons d'entrée matrice inférieurs à $2 \mathrm{~mm}$. Cette divergence n'a pas pu être 
Effort d'emboutissage $F$ (daN)

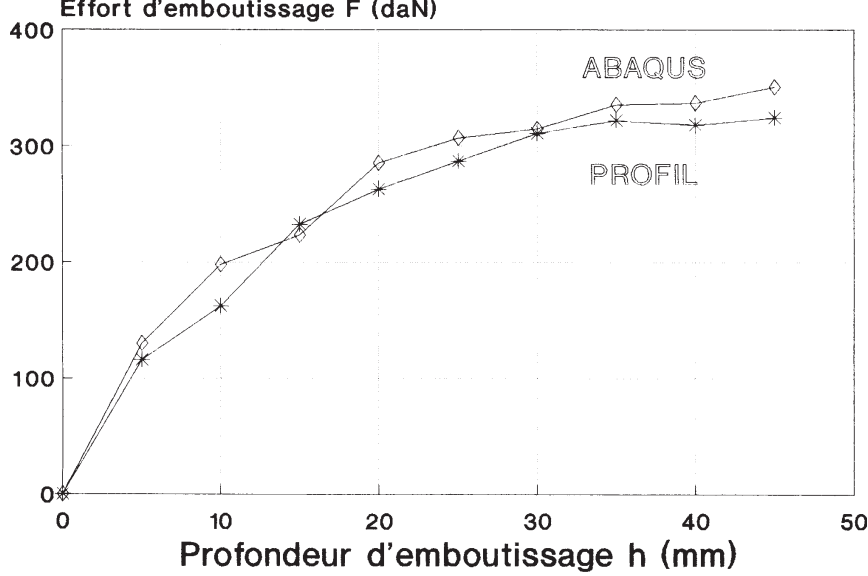

Fig. 17. Variation de l'effort d'emboutissage $F$ en fonction de la profondeur $h$.

surmontée même en réduisant le pas d'incrémentation et la taille des éléments au maximum.

\section{Conclusion}

Les résultats fournis par le logiciel «PROFIL» sont jugés très satisfaisants quand il s'agit d'emboutissage avec un grand rayon de matrice. Ils sont en parfait accord avec les résultats expérimentaux, ceux d'ABAQUS (écart inférieur à $10 \%$ pour les valeurs des rayons résiduels et les efforts d'emboutissage). Cependant, quand le rayon de matrice devient petit (inférieur à $3 \mathrm{~mm}$ ), les résultats du logiciel «PROFIL» ne suivent plus les résultats expérimentaux car l'hypothèse de la contrainte plane adoptée par ce logiciel n'est plus valable dans ce cas et la contrainte normale ne pourra plus être négligée.

Contrairement au logiciel «ABAQUS», le programme «PROFIL » est très rapide en exécution et aussi très facile d'utilisation car il est muni d'un programme de mise en données «MAILFLA $»$ interactif et simple d'accès, ce qui ne limite son utilisation à des personnes qualifiées et hautement spécialisées dans le domaine des éléments finis.

\section{Références}

[1] C.C. Chu, Elastic plastic springback of sheet metals subjected to complex plane strain bending histories, Solids Structures 22 (1986) 1071-1081
[2] M. Boivin, Prévision du retour élastique lors du pliage des tôles, Physique et Mécanique de la Mise en Forme des Métaux, $5^{\mathrm{e}}$ partie, Chapitre 9, Presses du C.N.R.S., Paris, 1990, pp. 471-480

[3] J.C. Boyer, M. Boivin, Calcul du retour élastique et des contraintes résiduelles lors du pliage des tôles sous tension, Grandes Déformations et Endommagement, Rapport GRECO No. 83, Laboratoire de Mécanique et Technologie, Cachan, France, 1983, 27 pages

[4] M. Akrout, Contribution à l'étude par éléments finis du retour élastique des tôles en emboutissage et déformation plane, Thèse de doctorat, Laboratoire Mécanique des Solides, INSA de Lyon, 1994, 194 p.

[5] L. Grisard, Modélisation bidimensionnelle de la mise en forme de tôles par la méthode des éléments finis, Thèse de doctorat, Université de Liège, 1992, 215 p.

[6] N.M. Wang, S.C. Tang, Analysis of bending effects in sheet forming operations, Intern. J. Numerical Methods in Engineering 25 (1988) 253-267

[7] M. Akrout, Modélisation de l'emboutissage de tôles et calcul du retour élastique par éléments finis, Mémoire de DEA Mécanique, INSA de Lyon, Villeurbanne, 1990

[8] C. Teodosiu, H.L. Cao, T. Ladreyt, J.M. Detraux, Implicit versus explicit methods in the simulation of sheet metal forming, Finite Element Simulation of 3D Sheet Metal Forming Processes in Automotive Industry, Zurich, 1991, pp. 259-291

[9] R.M. Mc Meeking, J.R. Rice, Finite element formulations for problems of large elastic-plastic deformation, J. solids and structures 11 (1975) 601-608

[10] M. Akrout, M. Brunet, Emboutissage des tôles minces et calcul du retour élastique par éléments finis, $11^{\mathrm{e}}$ Congrès Français de Mécanique, Lille, Villeneuve d'Ascq, 6-10 Septembre 1993, 4, pp. 57-60

[11] M. Brunet, Modélisation numérique des grandes déformations élasto-plastiques avec contact et frottement : Application à des problèmes spécifiques de la mise en forme des métaux, Thèse de doctorat d'état es-sciences, INSA de Lyon et Université Claude Bernard, 1987, $202 \mathrm{p}$.

[12] M. Brunet, Verification of simulation with experiment, NUMISHEET'93, 2nd International Conference Numerical Simulation of 3D Sheet Metal Forming Process, Tokyo Japon, 31 août-2 septembre 1993, pp. 647-652

[13] M. Brunet, A finite element method for unilateral contact and friction problem involving finite strain and large displacement, J. Theoretical and Applied Mechanics 7 (1988) 209-220

[14] M. Akrout, Validation du logiciel d'éléments finis PROFIL pour l'emboutissage des tôles minces, $1^{\mathrm{e}}$ colloque des doctorants, INSA Lyon, Villeurbanne, 21 avril 1994, pp. 61-62 